\title{
Sudden Cardiac Arrest Postoperative Day due to Pulmonary Embolism
}

\author{
Yeo-UI Yun, Sang-Min Shim, Yun-Sook Kim \\ Department of Obstetrics and Gynecology, Soonchunhyang University Cheonan Hospital, Soonchunhyang University College of Medicine, Cheonan, Korea
}

\begin{abstract}
Cardiac arrest one day after cesarean section is extremely rare. Obstetrical clinicians have low experience to these serious situations necessitating immediate first aid and knowledge of its differential diagnosis. A 33-year-old woman underwent elective repeat cesarean section at 38 weeks of gestation under spinal anesthesia. The patient underwent uneventful course on that day. Loss of consciousness occurred one day after cesarean section during her first ambulation. Immediate cardiac compression was performed and eventually resulted in good recovery of her heartbeat. Her condition was suitable disseminated intravascular coagulation (DIC). She developed acute ischemic pancreatitis after cardiac arrest. We describe the consideration of amniotic fluid embolism with DIC as most appropriate in this case. To our knowledge, our case is one of the most dangerous conditions after the cesarean section. Here, we report our case with a review of literatures.
\end{abstract}

Keywords: Cesarean section; Heart arrest; Pulmonary embolism; Pancreatitis

\section{INTRODUCTION}

The frequency of occurrence of cardiac arrest after delivery is extremely rare. Our patient occurred sudden cardiac arrest following loss of consciousness one day after cesarean section during her first ambulation. Most likely diagnosis was amniotic fluid embolism with disseminated intravascular coagulation (DIC). Because her condition and the results of coagulation profiles were suitable DIC. She developed acute ischemic pancreatitis in the process of recovery. We treated her supportive measures and oral anticoagulant micronized rivaroxaban tablet. The patient achieved a complete resolution of the embolism without recurrent episodes.

\section{CASE REPORT}

A healthy 33-year-old female, with a normal antenatal course, underwent the uneventful cesarean section in a primary hospital at 38 weeks of gestation. The next day, she developed a sudden loss of consciousness and a low blood pressure of 70/50 $\mathrm{mm} \mathrm{Hg}$, and was transferred to Soonchunhyang University Cheonan Hospital for precise diagnosis and treatment. On the way to our hospital emergency room, she had stupor mental state and no pulse rate. So, immediate cardiopulmonary resuscitation (CPR) was performed by physician of that clinic in the ambulance. On admission, her blood pressure was unable to be checked. And her electrocardiogram was showed a pulseless electrical activity. After five minutes of CPR, her cardiac rhythm was changed to a normal sinus rhythm. We had tracheal intubation, infusion of crystalloids and vasopressors, blood component replacement, and other supportive measures. Emergency contrast enhanced chest computed tomography (CT) showed a pulmonary embolism on bilateral interlobar pulmonary arteries and segmental branches of both lower lobes (Fig. 1). It also showed Interlobular septal thickening in both upper lobes and ground glass opacity in right upper lobe. On her laboratory test, serum electrolyte levels were sodium $142 \mathrm{mEq} / \mathrm{L}$ (reference range, 136 to $145 \mathrm{mEq} / \mathrm{L}$ ), chloride $112 \mathrm{mEq} / \mathrm{L}$ (reference range, 96 to $110 \mathrm{mEq} / \mathrm{L}$ ), and potassium $3.5 \mathrm{mEq} / \mathrm{L}$ (reference range, 3.5 to $5.1 \mathrm{mEq} / \mathrm{L}$ ). Urine analysis showed $>100$ red blood cells, $>100$ white blood cells, proteinuria +3 , trace ketones, $1-4 \mathrm{ep-}$ ithelial cells, and $<1$ casts per microscopic examination. The val-
Correspondence to: Yun-Sook Kim

Department of Obstetrics and Gynecology, Soonchunhyang University Cheonan Hospital, Soonchunhyang University College of Medicine,

31 Suncheonhyang 6-gil, Dongnam-gu, Cheonan 31151, Korea

Tel: +82-41-570-2150, Fax: +82-41-571-7887, E-mail: drsook@schmc.ac.kr

Received: Jun. 12,2015 / Accepted after revision: Jul. 16, 2015
(C) 2015 Soonchunhyang Medical Research Institute This is an Open Access article distributed under the terms of the Creative Commons Attribution Non-Commercial License (http://creativecommons.org/licenses/by-nc/3.0/). 


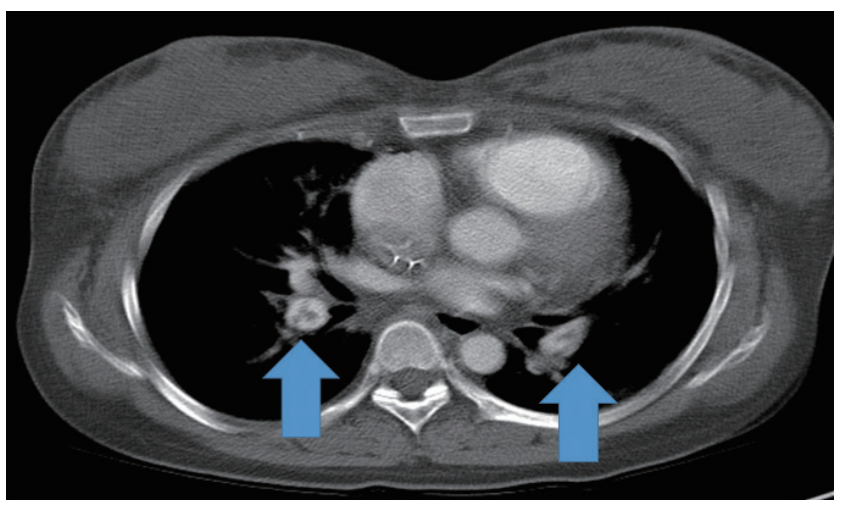

Fig. 1. Contrast enhanced chest CT scans. The chest CT scans on her admission day showed severe motion artifact, but multiple pulmonary embolism bilateral interlobar pulmonary arteries (arrow) and segmental branches of both lower lobes, interlobular septal thickening in both upper lobes and ground glass opacity in right upper lobe and left lung. CT, computed tomography.

ues of arterial blood gas analysis were $\mathrm{pH} 6.91$ (reference range, 7.35 to 7.45), $\mathrm{pCO}_{2} 45 \mathrm{~mm} \mathrm{Hg}$ (reference range, 32.0 to $45.0 \mathrm{~mm}$ $\mathrm{Hg}$ ), bicarbonate $9.0 \mathrm{mmol} / \mathrm{L}$, and base excess $-23.7 \mathrm{mmol} / \mathrm{L}$. Hemoglobin was $9.3 \mathrm{~g} / \mathrm{dL}$ (reference range, 12.0 to $16.0 \mathrm{~g} / \mathrm{dL}$ ), platelet $103 / \mathrm{mm}^{3}$ (reference range, 130 to $400 / \mathrm{mm}^{3}$ ), and C-reactive protein $30.28 \mathrm{mg} / \mathrm{L}$ (reference range, 0.01 to $3.0 \mathrm{mg} / \mathrm{L}$ ). The coagulation profiles were prothrombin time 22.5 seconds (reference range, 9.8 to 12.4 seconds), activated partial thromboplastin time $28.7 \%$ (reference range, $80 \%$ to $135 \%$ ), and international normalized ratio 2.03 (reference range, 0.86 to 1.10). Hepatitis B antigen, syphilis, and human immunodeficiency viruses were all negative. Blood urea nitrogen was $3.8 \mathrm{mg} / \mathrm{dL}$ (reference range, 8.0 to $20 \mathrm{mg} / \mathrm{dL}$ ), creatinine $0.8 \mathrm{mg} / \mathrm{dL}$ (reference range, 0.5 to $1.2 \mathrm{mg} / \mathrm{dL}$ ), uric acid $6.2 \mathrm{mg} / \mathrm{dL}$ (reference range, 3.0 to $7.0 \mathrm{mg} / \mathrm{dL}$ ), total bilirubin 0.1 $\mathrm{mg} / \mathrm{dL}$ (reference range, 0.2 to $1.2 \mathrm{mg} / \mathrm{dL}$ ), alanine aminotransferase $413 \mathrm{IU} / \mathrm{L}$ (reference range, 0 to $40 \mathrm{IU} / \mathrm{L}$ ), aspartate aminotransferase $266 \mathrm{IU} / \mathrm{L}$ (reference range, 0 to $40 \mathrm{IU} / \mathrm{L}$ ), lactate dehydrogenase $567 \mathrm{IU} / \mathrm{L}$ (reference range, 0 to $250 \mathrm{IU} / \mathrm{L}$ ), creatine kinasemyocardial band $79 \mathrm{U} / \mathrm{L}$ (reference range, 0 to $24 \mathrm{U} / \mathrm{L}$ ), troponin-T $0.144 \mathrm{ng} / \mathrm{mL}$ (reference range, 0.000 to $0.100 \mathrm{mg} / \mathrm{mL}$ ), amylase 40 IU/L (reference range, 8 to $110 \mathrm{IU} / \mathrm{L}$ ), lipase $28 \mathrm{U} / \mathrm{L}$ (reference range, 0 to $60 \mathrm{U} / \mathrm{L}$ ), D-dimer $49.9 \mu \mathrm{g} / \mathrm{mL}$ (reference range, 0 to 0.5 $\mu \mathrm{g} / \mathrm{mL}$ ), fibrinogen degradation product $80 \mu \mathrm{g} / \mathrm{mL}$ (reference range, 0 to $0.5 \mu \mathrm{g} / \mathrm{mL}$ ), and fibrinogen $124.5 \mathrm{mg} / \mathrm{dL}$ (reference range, 180 to $450 \mathrm{mg} / \mathrm{dL}$ ). The results of her coagulation profiles were suitable DIC.

We considered a pulmonary thromboembolism or an amniotic fluid embolism with DIC. She was admitted to an intensive care
Table 1. Serial measurements of amylase and lipase levels

\begin{tabular}{lcc}
\hline Hospital day & Amylase (8-110 IU/L) & Lipase (0-60 U/L) \\
\hline Admission day & 40 & 28 \\
1 & 198 & 52 \\
2 & 265 & 489 \\
3 & 314 & 601 \\
5 & 267 & 584 \\
9 & 185 & 450 \\
11 & 210 & 393 \\
13 & 143 & 226 \\
\hline
\end{tabular}

unit. Half dose of heparin was injected to her to dissolve embolism, and also she was given an intravenous administration of 5 mg sodium bicarbonate to correct the respiratory acidosis. After we gave her heparin injection, there was much amount of bleeding from her operation wound with lowering blood pressure. Promptly we stopped heparin infusion. Two days after admission, her vital sign was normalized and no more bleeding. She was successfully extubated within 48 hours. We treated her oral anticoagulant micronized rivaroxaban tablet $30 \mathrm{mg}$ a day. Fortunately, her vital sign was stable, and on follow-up chest CT scan, embolism seems to be slightly decreased. But on her follow-up laboratory test on admission day 3 was elevated serum amylase 198 IU/L and lipase 52 U/L. The hepatologist regarded her as acute ischemic pancreatitis after CPR. We also checked her abdominal ultrasonography, there was pancreatic enlargement and gallbladder wall edema. We put her nothing per oral and gave an intravenous administration of antibiotics such as cefepime $2 \mathrm{~g}$ and metronidazole $1.5 \mathrm{~g}$ a day because of anerobes empirically for 10 days. We did daily inspection of her amylase and lipase (Table 1). She started eating soft diet on admission day 11. The levels of amylase and lipase were still high, but these seemed to be stabilized. On hospitalization day 17, the patient was discharged from us after receiving micronized rivaroxaban tab $30 \mathrm{mg}$ a day without neurological and cardiovascular complications. One week later, the patient received follow-up laboratory examinations. This showed that the patient achieved a recovery of pancreatic function. Two months thereafter, the patient stopped medication and achieved a complete resolution of the embolism without recurrent episodes. In addition, at 12-month follow-up, the patient had no notable findings or recurrences.

\section{DISCUSSION}

The frequency of occurrence of cardiac arrest in pregnancy is 1 
in 30,000 during pregnancy, of which even much rarer postoperative [1]. There are multiple causes of cardiac arrest after delivery, obstetric and non-obstetric. According to the World Health Organization, pulmonary embolism is the worlds' third cause of maternal mortality. The origin of the embolus can have different, such as arterial and venous thrombotic embolisms, or amniotic fluid embolism. The differentiation of thromboembolism and amniotic fluid embolism is very difficult and often a definitive diagnosis can only be made at autopsy [2]. The chest CT with pulmonary angiography is currently the most commonly employed technique used for pulmonary embolism diagnosis.

The amniotic fluid syndrome was described in 1941 by Steiner and Lushbaugh and became classically characterized by the abrupt onset of hypotension, hypoxia, and severe consumptive coagulopathy [3]. The amniotic fluid embolism can be confirmed by histological confirmation of amniotic fluid contents in the pulmonary vasculature of the mother, although difficult to identify some contests in the small pulmonary capillaries. The estimated incidence of amniotic fluid embolism is 2.0 per 100,000 deliveries. The risk factors were induction of labor, multiple pregnancy, elderly women, and cesarean delivery [4]. The mechanism of amniotic fluid embolism is thought to the result of maternal anaphylactic reaction to the fetal material entering the maternal pulmonary circulation. That can cause apnea, hypotension, loss of consciousness, cardiac arrest, and even the maternal death. In the first stage, fetal cells and amniotic fluid enter the maternal circulation. These triggers the secretion of the various endogenous substances. In the second stage, the spasm of pulmonary artery leads to increase right ventricular pressure, and maternal hypoxia as a result. The hypoxia causes maternal myocardial and pulmonary capillary damages. Roughly, half of all patients who survive enter a second stage was characterized by bleeding and DIC due to low level of fibrinogen. Because the amniotic fluid contains much amount of the thromboplastin [5]. There is no standard definite diagnostic examination. Therefore, it is a diagnosis of exclusion. Benson [6] proposed a clinically based definition of amniotic fluid embolism. According to his criteria, a diagnosis of amniotic fluid embolism would be appropriate for any patient experiencing sudden onset of cardiovascular collapse during pregnancy or 48 hours postpartum [6]. Treatment is supportive. Tracheal intubation, cardiopulmonary resuscitation, and other supportive measures must be instituted without delay. Treatment is directed at oxygenation and support of the failing myocardium, along with circulatory support that includes rapid blood and component replacement [7].

In this case, we could not histologic confirmation. We considered a pulmonary thromboembolism or an amniotic fluid embolism. But the results of her coagulation profiles were suitable DIC. We describe the consideration of amniotic fluid embolism with DIC as most appropriate in this case. So we had tracheal intubation, infusion of crystalloids and vasopressors, blood component replacement, and other supportive measures.

Venous air embolism is the entrapment of air into the venous system causing symptoms and signs of pulmonary vessel obstruction. It has been associated with cesarean section, especially in case of placenta previa. Since transesophageal echocardiography showed no sign of venous air embolism, this diagnosis seems unlikely [8].

The acute ischemic pancreatitis can be occurred after cardiac arrest. The diagnosis of acute pancreatitis was made on the association of abdominal pain with increased amylase and lipase concentrations. Until now, there is no consensus for recommending levels of plasma amylase and lipase concentrations after cardiac arrest [9].

In our case, it is hard to rule out thromboembolism or amniotic fluid embolism, so we manage both disease together. And we treated her with anticoagulant and supportive care together, and eventually resulted in good recovery. So if it is hard to differentiate the origin of embolism, it will be good choice to take care of both disease together. Moreover, plasma amylase and lipase concentrations should be measured after cardiac arrest in patients presenting with digestive symptoms, like as abdominal bloating, ileus or feeding difficulty.

\section{REFERENCES}

1. Whitty JE. Maternal cardiac arrest in pregnancy. Clin Obstet Gynecol 2002;45:377-92.

2. Ecker JL, Solt K, Fitzsimons MG, MacGillivray TE. Case records of the Massachusetts General Hospital: case 40-2012: a 43-year-old woman with cardiorespiratory arrest after a cesarean section. N Engl J Med 2012;367:2528-36.

3. Ihara K, Naito S, Okado T, Rai T, Mori Y, Toda T, et al. Successful recovery from an acute kidney injury due to amniotic fluid embolism. Intern Med 2015;54:49-54.

4. Knight M, Tuffnell D, Brocklehurst P, Spark P, Kurinczuk JJ; UK Obstetric Surveillance System. Incidence and risk factors for amniotic-fluid embolism. Obstet Gynecol 2010;115:910-7.

5. Van Liempt SW, Stoecklein K, Tjiong MY, Schwarte LA, de Groot CJ, Teunissen PW. Essentials in cardiac arrest during cesarean section. Clin Pract 2015;5:668.

6. Benson MD. A hypothesis regarding complement activation and amni- 
otic fluid embolism. Med Hypotheses 2007;68:1019-25.

7. Mahshid N, Ahmad S, Nahid M, Afshin F. Sudden cardiac arrest during cesarean section: a possible case of amniotic fluid embolism. Middle East J Anaesthesiol 2009;20:315-7.

8. Marshall AL. Diagnosis, treatment, and prevention of venous thrombo- embolism in pregnancy. Postgrad Med 2014;126:25-34.

9. Piton G, Barbot O, Manzon C, Moronval F, Patry C, Navellou JC, et al. Acute ischemic pancreatitis following cardiac arrest: a case report. JOP 2010;11:456-9. 